\title{
A Full Duplex D2D Clustering Resource Allocation Scheme Based on a K-Means Algorithm
}

\author{
Xu Huang $₫$, ${ }^{1,2}$ Mengjia Zeng, ${ }^{1}$ Jing Fan, ${ }^{2}$ Xiangxiang Fan, ${ }^{1}$ and Xuefeng Tang ${ }^{1}$ \\ ${ }^{1}$ School of Information Engineering, Huzhou University, Huzhou, Zhejiang 313000, China \\ ${ }^{2}$ College of Control Science and Engineering, Zhejiang University, Hangzhou, Zhejiang 310058, China \\ Correspondence should be addressed to Xu Huang; hx@zjhu.edu.cn
}

Received 5 March 2018; Accepted 22 April 2018; Published 23 May 2018

Academic Editor: Liu Liu

Copyright (c) $2018 \mathrm{Xu}$ Huang et al. This is an open access article distributed under the Creative Commons Attribution License, which permits unrestricted use, distribution, and reproduction in any medium, provided the original work is properly cited.

\begin{abstract}
Although the Device-to-Device (D2D) technology in cellular networks can improve the performance of cellular systems, it creates a large amount of interference in traditional communications. In this paper, the problem of resource allocation and control in a single-cell scene is studied. First, the concept of a restricted D2D communication area and a restricted D2D user-reusage area is put forward to reduce the complexity and interference intensity of resource allocation. Second, under the premise of satisfying the QoS (Quality of Service) demands of every system user, the resource allocation algorithm is improved, the optimal allocation of resources is carried out, and the algorithm's processes are given in detail. Our simulated experiments show that the proposed method greatly improves the spectrum efficiency and the system fairness.
\end{abstract}

\section{Introduction}

In recent years, the rise and rapid development of smart phones have profoundly changed the way we communicate and enjoy digital entertainment. The wide use of wireless applications, such as cloud computing, surfing the Internet, and downloading and watching digital multimedia, has created a large demand for high-speed and efficient wireless communication technology. $5 \mathrm{G}$ is the next-generation mobile communication system that is being developed for the expected demand of information and communication after 2020. It will have higher spectrum utilization and transmission rate, significantly improved transmission delay and QoS (Quality of Service) perception, and an increased number of access links and security [1].

Device-to-Device (D2D) technology $[2,3]$ is a hot topic in the field of mobile communication. Using D2D technology, adjacent terminals can transmit data within a close range through a direct link without a central node. In this case, the base station sends only some control information, which greatly reduces the load of the base station $[2,4,5]$. D2D users can effectively reuse the wireless resources authorized by the network to enhance the reusage rate of the wireless spectrum and expand the throughput and coverage of heterogeneous cellular networks [6, 7]. In full duplex mode, the spectrum benefit is doubled as a result of allowing users to synchronize and send and receive signals simultaneously $[8,9]$. This technology can greatly improve the wireless transmission rate, and it has great technical advantages and application prospects. As such, it is a promising option for future 5G communication.

However, under the same cell, channel resources are reused by $\mathrm{D} 2 \mathrm{D}$ communication and cellular users simultaneously. So, while D2D communication technology brings convenience, it also results in frequency interference [10]. As the number of users increases, the interference between them also rises. The problem of network capacity optimization and power allocation also arises, which leads to an increase in the power consumption of the entire system. In heterogeneous networks, there are two resource allocation schemes: one is local resource allocation (fixed-cell user resource and adaptive resource allocation for a D2D pair) and the other is global resource allocation (the cell service user and the D2D pair are allocated resources jointly) [11]. Because D2D communications can compete and cooperate with each other to share resources, their individual or group behaviors conform to the inherent nature of game theory, which allows them to be effectively modeled and analyzed [12]. 
The combination of D2D communication technology and a cognitive radio (CR) can effectively reduce interference [13]. CR technology, through interactions with the external environment in terms of multidimensional spectrum detection, as well as real-time and interactive environments, is able to perceive any interference and make subsequent judgments so that cognitive users can choose the most appropriate communication frequency to avoid interference to primary users under the condition of the spectrum with the primary users sharing [14]. How to manage the spectrum resources of the community as a whole, reasonably determine the communication power of each device, and minimize the interference between devices have become the main bottleneck for D2D communication to enter the practical stage. It is worthwhile to try to achieve power stability $[15,16]$ or learn from software reliability prediction through context sensitive rate Boolean control network [17].

Considering the strengths and weaknesses of current D2D communication research, we have studied resource allocation and power control in the single-cell scenario based on the work of [18]. First, the concept of a restricted D2D communication area and a restricted D2D usage area is put forward to reduce the complexity and interference intensity of resource allocation. Second, under the premise of satisfying the QoS of all users in the system, the resource allocation algorithm is improved, the optimal allocation of resources is carried out, and a detailed description of the algorithm's processes is given. Our simulated experiments show that the proposed method greatly improves the spectrum efficiency and system fairness.

\section{Problem Description}

For a single-cell model, it is assumed that the cell includes $M$ cellular users and $N$ D2D user pairs, and the number of subchannels in this cell is $L$. The set of cellular users is $C=\left\{C_{1}, C_{2}, \ldots, C_{M}\right\}$, while the set of D2D user pairs is $D=$ $\left\{D_{1}, D_{2}, \ldots, D_{N}\right\}$. D2D user pairs can reuse the cellular users' uplink resources. Compared with a user device, the base station has stronger anti-interference ability and processing power, and the amount of data in the cellular network is asymmetric, where the uplink resources are not fully utilized $[19,20]$. Therefore, the D2D communication discussed in this paper chooses to reuse the uplink resources of the cellular system.

Assuming that the location coordinates of each user device are known to the base station, the channel gain between any users and between the users and the base stations can be calculated. In a cellular system model, BS is the base station, and the $i$ th $\mathrm{D} 2 \mathrm{D}$ user pair $\mathrm{D}_{2} \mathrm{D}_{i}$ reuses the uplink resource of the $j$ th cellular user. See Figure 1.

As shown in Figure 1, $\mathrm{CU}_{j}$ send signals to the BS, where the channel gain is $g_{j, B}$. The transmitter $\mathrm{D}_{2} \mathrm{D}_{i, t x}$ of the $i$ th D2D pairs $\mathrm{D}_{2} \mathrm{D}_{i}$ sends signals to the receiver $\mathrm{D}^{2} \mathrm{D}_{i, r x}$, where the channel gain is $g_{i}$. When $\mathrm{CU}_{j}$ transmits a signal, there

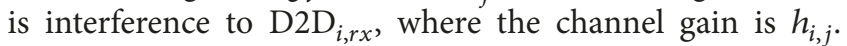
Additionally, there are interferences to the base station when D2 $D_{i, t x}$ transmits a signal, where the channel gain is $h_{i, B}$ and the Gaussian white noise is $N_{0}$.

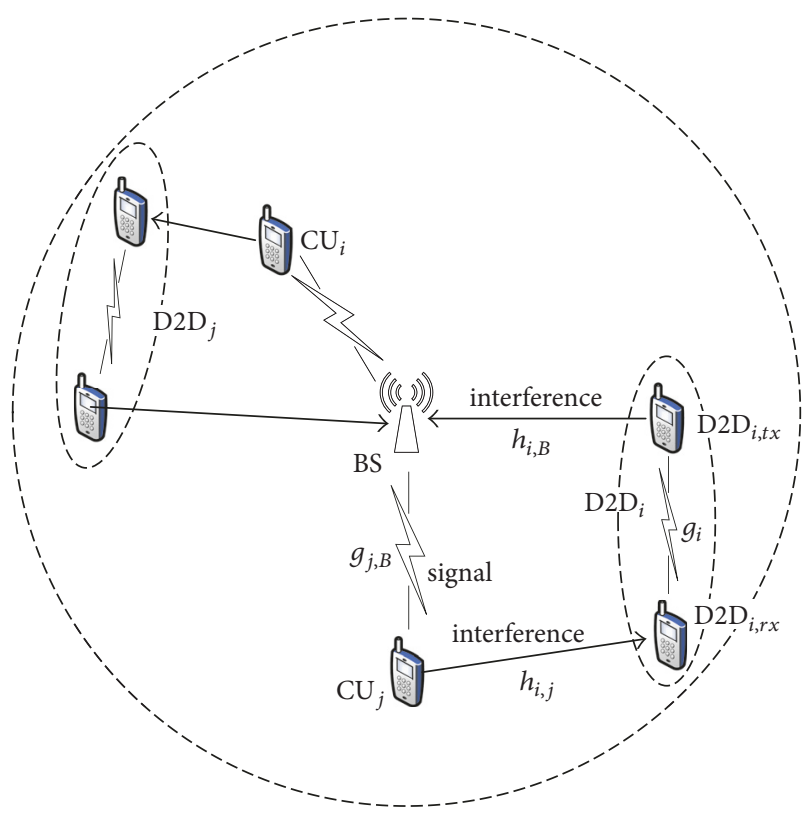

Figure 1: System model.

Regarding the road-loss model, the slow fading caused by the multipath effect and the fast fading caused by the shadow effect should also be considered. As such, the channel gains of the D2D pairs' transmitter $\mathrm{D}_{2} \mathrm{D}_{i, t x}$ to the base station and

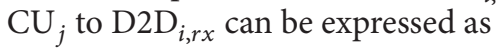

$$
\begin{aligned}
& h_{i, B}=K \cdot \delta_{i, B} \cdot \xi_{i, B} \cdot d_{i, B}^{-\alpha}, \\
& h_{i, j}=K \cdot \delta_{i, j} \cdot \xi_{i, j} \cdot d_{i, j}^{-\alpha} .
\end{aligned}
$$

Among them, $K$ is the road-loss constant and $\alpha$ is the road loss index, which are both determined by the cellular system environment. $d_{i, B}$ is the distance from $\mathrm{D} 2 \mathrm{D}_{i, t x}$ to the base

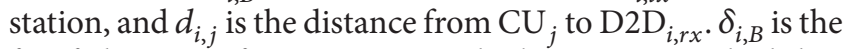
fast fading gain from $\mathrm{D} 2 \mathrm{D}_{i, t x}$ to the base station, which has an exponential distribution, $\delta_{i, j}$ is the fast fading gain from

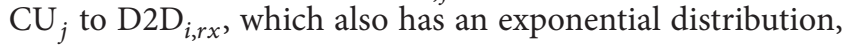
$\xi_{i, B}$ is the slow fading gain from $\mathrm{D}_{2} \mathrm{D}_{i, t x}$ to the base station, which has a logarithmic distribution, and $\xi_{i, j}$ is the slow fading gain from $\mathrm{CU}_{j}$ to $\mathrm{D}_{2} \mathrm{D}_{i, r x}$, which also has a logarithmic distribution.

The foregoing description shows that there are three kinds of interference in the considered scenario: (1) interference from the D2D transmitter to the cellular system; (2) interference from the cellular users to the D2D receiver; and (3) interference between D2D pairs sharing the same spectrum resources. The problem to be solved in this paper is to establish a cellular resource reuse state system model under the consideration of all three kinds of interference and use it to determine the best resource allocation plan.

\section{Resource Allocation Scheme Satisfying System QoS}

3.1. D2D User Pairs Delimit Communication Restricted Areas. As a D2D user reuses the upstream resource of a cellular user, 


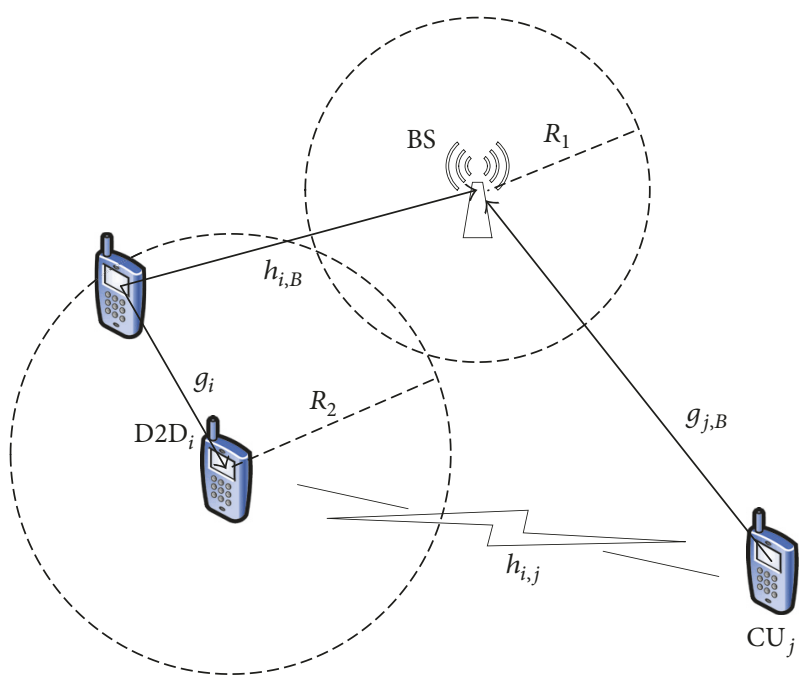

FIGURE 2: Cellular users in relation to the limited D2D communication area and the reusage area.

the closer the $\mathrm{D} 2 \mathrm{D}$ user is to the base station, the greater the amount of interference is imparted to the $\mathrm{BS}$ receiving the $\mathrm{CU}$ (cellular user) signals. Therefore, to reduce the interference, we propose an improvement to the total throughput of the system while also reducing the computation complexity; we suggest restricting the size of the D2D communication area. In this area, D2D is considered to contribute a large amount of interference to the base station. Out of this area, any interference from D2D to the base station can be ignored. Assuming that $P_{\max }^{d}$ is the maximum transmission power of all D2D users, $I_{d, B}$ indicates the interference threshold value of all D2D users to the base station, which must satisfy

$$
I_{d, B} \geq P_{\max }^{d} \cdot h_{i, B} .
$$

The interference of all D2D users to the base station must be less than this threshold value; otherwise, it will cause more serious interference to the base station, so that cellular users cannot communicate effectively. Among them, $I_{d, B}$ is obtained via long-term observations of a cellular cell. By combining (1) and (3), we can solve for the radius $R_{1}$, which is the radius of $\mathrm{D} 2 \mathrm{D}$ communication limited area:

$$
d_{i, B} \geq\left[\frac{P_{\max }^{d} \cdot K \cdot \delta_{i, B} \cdot \xi_{i, B}}{I_{d, B}}\right]^{\alpha^{-1}}=R_{1} .
$$

Therefore, the limited D2D communication area is a circular area of radius $R_{1}$ with the BS at the center (see Figure 2). When the base station receives a request to establish a D2D link, the base station first determines whether the D2D transmitter is in the D2D communication area, and if it is, then this prohibits the establishment of D2D link.

3.2. Cellular Users Are Restricted to the Reusage Area. We can see from Figure 1 that when D2D users and cellular users use the same uplink resources, there will be interference from $\mathrm{CU}_{j}$ to $\mathrm{D} 2 \mathrm{D}_{i, r x}$, where the closer the distance between them is, the greater the interference will become. Therefore, we propose to delineate a restricted reusage area for the cellular users to reduce this kind of interference. Assuming that $P_{\max }^{c}$ represents the maximum transmit power of the cellular users, $I_{c, d}$ represents the threshold value of the cellular users' interference to the D2D users. Hence, the total imparted interference from all cellular users to the D2D users must be less than this threshold value; otherwise the D2D users cannot communicate normally. That is to say, the following formula should be satisfied:

$$
I_{c, d} \geq P_{\max }^{c} \cdot h_{i, j} \cdot
$$

Among them, $I_{c, d}$ is determined by long-term observations of cellular cells. If we combine (2) and (5), we can calculate the restricted area radius $R_{2}$ of the cellular users:

$$
d_{i, j} \geq\left[\frac{P_{\max }^{c} \cdot K \cdot \delta_{i, j} \cdot \xi_{i, j}}{I_{c, d}}\right]^{\alpha^{-1}}=R_{2} .
$$

So, the limited, reusage area for a cellular user is a circle with radius $R_{2}$, where $\mathrm{D} 2 \mathrm{D}_{i, r x}$ is located at the center (Figure 2). Any cellular users in this area are not selected as potential reusage objects by any $\mathrm{D} 2 \mathrm{D}$ pairs.

3.3. System Model Establishment. To satisfy all users QoS requests, we must ensure that the D2D users and cellular users reach their respective minimum SINR (signal-tointerference-plus-noise ratio). For device $K$ in the cellular $m$, the SINR of the device when communicating is

$$
\operatorname{SINR}(m, k)=\frac{P_{R}(m, k)}{I(m, k)+P_{n}}, \quad \forall m, 1 \leq m \leq M .
$$

Among them, $P_{R}(m, k)=P_{T}(m, k) \cdot G_{T}(m, k)$ indicates the valid signal strength that device $K$ receives from cellular $m$, $P_{T}(m, k)$ is the transmission power of each base station RB (resource block), $G_{T}(m, k)$ is the channel gain between the BS and the device, and $I(m, k)$ is the summed interference from all other devices.

The goal is to maximize the system's throughput:

$$
\begin{array}{ll}
\max & \left(\sum_{i=1}^{N} \log _{2}\left(1+\gamma_{\mathrm{CU}_{j}}\right)+\sum_{j=1}^{K} \log _{2}\left(1+\gamma_{\mathrm{DU}_{i}}\right)\right) \\
\text { s.t. } & \gamma_{\mathrm{CU}_{j}}=\frac{P_{j}^{c} h_{c j, B}}{\sum_{i=1}^{k} P_{i}^{d} h_{d i, c j} x_{i, j}+N_{0}} \geq \mathrm{SINR}_{\min }^{c}, \\
& \gamma_{\mathrm{DU}_{i}} \\
= & \frac{P_{i}^{d} h_{d i, d i}}{\sum_{j=1, l \neq i}^{k} P_{l}^{d} h_{d l, d i} x_{l, i}+\sum_{j=1}^{N} P_{j}^{c} h_{c j, d i} x_{i, j}+N_{0}} \\
\geq \operatorname{SINR}_{\min }^{d}, \quad \forall i \in D, \\
0 \leq P_{j}^{c} \leq P_{\max }^{c}, \quad \forall i \in C, \\
0 \leq P_{i}^{d} \leq P_{\max }^{d}, \quad \forall j \in C .
\end{array}
$$


Among them, $\gamma_{\mathrm{CU}_{j}}$ represents the actual SINR of the cellular users, and $\gamma_{\mathrm{DU}_{i}}$ represents the actual SINR of the D2D users. $P_{j}^{c}$ and $P_{i}^{d}$, respectively, represent the actual transmission power of the cellular users $j$ and the D2D users $i$, while $h_{c j, B}$ indicates the channel gain between the cellular users $j$ and the base station. The model is $128.1+37.6 \cdot \lg D$, where $D$ is the distance between the cellular users and the BS in units of Km. $h_{d i, d i}, h_{d l, d i}, h_{c j, d i}$, and $h_{d i, c j}$, respectively, represent the channel gain between users, where the model is $10 \cdot \lg D 4$ and $D$ is the distance between two users in units of $m . x_{i, j}$ is a binary value: if users $i$ and $j$ share the same resources, $x_{i, j}=$ 1 ; otherwise $x_{i, j}=0$. Finally, $P_{\max }^{c}$ and $P_{\max }^{d}$, respectively, represent the minimum SINR of a cellular user and a D2D pair when they communicate normally.

The foregoing objective function represents the maximum throughput of the complete system. The first two restricted conditions (see (8a) and (8b)) ensure that cellular users and D2D users meet their QoS needs. Only those D2D users that satisfy these two conditions can be connected to the network at the same time. The last two restricted conditions (see $(8 \mathrm{c})$ and $(8 \mathrm{~d})$ ) indicate that the transmission power of the cellular and D2D users should not exceed the maximum transmission power.

3.4. D2D Pair Clustering to Solve the Problem of Interference between D2D Pairs and Each Other. In cellular communication systems, there is interference between D2D pairs and each other. To effectively use the available system resources, it is necessary to reduce this kind of interference by grouping D2D pairs into clusters. It is considered that, in the same cluster, the interference between D2D pairs and each other can be ignored, and it is possible to reuse resources for the same CU. As their location coordinates are known, the distance between the D2D pairs can be calculated. The closer the distance between $\mathrm{D} 2 \mathrm{D}$ pairs is, the greater the interference becomes; that is, the distance between D2D pairs is inversely proportional to the interference value. The reciprocal of the distance value is used to represent of the interference, where a matrix of interference values between the D2D pairs can be formed. Then, the $K$-means clustering method is used to divide the D2D clusters according to their interference value in the matrix. Assuming that the total number of D2D pairs is $N$, all D2D devices are grouped as $X=\{x(i), i=1,2, \ldots, N\}$. After clustering, $K_{C}$ clusters are formed and recorded as $C=$ $\left\{c(j), j=1,2, \ldots, K_{C}\right\}$. The specific algorithm process is as follows:

(1) Randomly select the initial cluster centers of $K_{C}$ as $U=$ $\left\{u(j), j=1,2, \ldots, K_{C}\right\}$.

(2) The distance between samples is calculated, and their interference value in the matrix is formed from the reciprocal of the distance value, and the sample is added to the cluster with the smallest interference value.

(3) Calculate the summed square distance between points in cluster $c(j)$ and the cluster center $u(j)$, as well as the total summed square distance:

$$
J[c(j)]=\sum_{x(i) \in c(j)}\|x(i)-u(j)\|^{2}
$$

$$
\begin{aligned}
J(C) & =\sum_{j=1}^{K_{C}} J[c(j)]=\sum_{j=1}^{K_{C}} \sum_{i=1}^{N_{U E}} d_{j i}\|x(i)-u(j)\|^{2}, \\
d_{j i} & = \begin{cases}1, & x(i) \in c(j) \\
0, & x(i) \notin c(j) .\end{cases}
\end{aligned}
$$

According to the least-square method and the Lagrange principle, the cluster center $u(j)$ takes the average of each sample point within the corresponding cluster $c(j)$.

(4) According to these steps, we iterate and update the cluster until $J(C)$ converges to a minimum value, whereby the iteration is complete.

After iteration, we output the centers of the $K_{C}$ clusters $\left\{u_{1}, u_{2}, \ldots, u_{K_{C}}\right\}$ and decide which D2D devices are included in each cluster.

3.5. Resource Allocation of the D2D User Cluster under a QoS Guarantee. Through the aforementioned steps, all D2D pairs are divided into $K$ clusters, each of which is called $g_{k}$. Because the interference arising from the D2D network is different for each TTI (Transmission Time Interval), the number and the size of the clusters are not fixed, which makes full use of the instantaneous channel state information of the D2D network. The main task of this section is to determine which subchannel is assigned to which cluster.

Let us consider the following model for resource sharing: the cellular users are allocated resources as a prior, and each user occupies a cellular RB. A single D2D cluster can reuse most of the cellular users' spectrum resources; otherwise, a cellular spectrum resource is only reused by a single D2D cluster. The channel allocation is represented by the matrix $Y=\left[y_{k n}\right]$. Element $y_{k n}=1$ indicates that channel $n$ is assigned to the D2D cluster $K$; otherwise, it is equal to 0 . The goal of this article is to find an allocation method $Y_{\text {opt }}$ to maximize the throughput of the whole system; that is,

$$
Y_{\mathrm{opt}}=\underset{Y \in J}{\arg \max } \sum_{K} \sum_{n} R_{k n} \cdot y_{k n}
$$

among which $R_{k n}$ represents the data rate of the cluster $K$ to the $\mathrm{RB}_{n}$. To ensure normal communication throughout the original cellular network, in the actual resource allocation stage, it is necessary to screen any D2D users not only meeting the D2D QoS request from each cluster and to also ensure the normal communication of all cellular users and grant them access to the network. Therefore, the $\mathrm{D} 2 \mathrm{D}$ pairs in the same cluster $g_{k}$ are different, which shared different resource blocks of the same cellular user. Cluster $g_{k j \text { Share }}$ is used to represent a D2D set that can share $\mathrm{RB}_{j}$ in cluster $g_{k}$.

For a particular RB, the screening process for the D2D users that can eventually access the network is as follows:

(1) Initialization: consider the case of a particular cluster $g_{k}$ sharing $\mathrm{RB}_{j}$, and create two new clusters of $g_{k j}$ and $g_{k j \text { Share }}$

(2) for $i=1: M(I, \mathrm{GK})$ do 
calculate the distance $d_{i, j}$ between $\mathrm{CU}_{j}$ and $\mathrm{D}_{2} \mathrm{D}_{i}$ on $\mathrm{RB}_{j}$

calculate the distance $d_{i, B}$ between $\mathrm{D}_{2} \mathrm{D}_{i}$ and the base station BS

calculating the $\mathrm{SINR}_{d i}$ of $\mathrm{D}_{2} \mathrm{D}_{i}$

if $\mathrm{D}_{2} \mathrm{D}_{i}$ satisfies $\mathrm{SINR}_{d i} \geq \operatorname{SINR}_{\min }^{d}$ and $d_{i, B} \geq R_{1}$ and $d_{i, j} \geq R_{2}$

put $\mathrm{D} 2 \mathrm{D}_{i}$ into cluster $g_{k j}$

end if

end for

(3) for $i=1: M\left(i \in g_{k j}\right)$ do

calculate the interference value of $\mathrm{D}_{2} \mathrm{D}_{i}$ to the cellular user in $\mathrm{RB}_{j}$

end for

Ascendingly sort the interference values of all D2D users in $g_{k j}$

(4) for D2D users after sorting do

calculate the SINR of the cellular user after added the $\mathrm{D} 2 \mathrm{D}$ pairs

if the SINR of the cellular user satisfies SINR $\geq$ $\mathrm{SINR}_{\min }^{c}$

\section{break}

end if

else

put this D2D user into cluster $g_{k j \text { Share }}$

end else

end for

Step (2) filters the D2D pairs in cluster $g_{k}$ by considering the interference between the D2D pairs and the base station and from the cellular users to the D2D users. If a QoS request cannot be fulfilled, we then obtain cluster $g_{k j}$. Step (3) calculates the interference value of each D2D pair in cluster $g_{k j}$ and, according to the interference value sorts the corresponding D2D users. Step (4) starts with the D2D pair that has the minimum value of interference from cellular users in cluster $g_{k j}$ and then calculates the SINR of the cellular user when this D2D pair is shared $\mathrm{RB}_{j}$. If the SINR is less than the minimum SINR of the cellular user, the system does not allow the D2D pair to share $\mathrm{RB}_{j}$; otherwise, it puts the D2D pair into cluster $g_{k j \text { Share }}$. The system then considers the D2D user with the second smallest interference value and calculates these cellular users' SINR. The entire process is repeated until every D2D pair in $g_{k j}$ has been calculated. Finally, all D2D users in cluster $g_{k j}$ which can share $\mathrm{RB}_{j}$ are obtained and are represented by the cluster $g_{k j \text { Share }}$.

In this paper, we use throughput ${ }_{R j}$ to characterize the throughput achieved by each cluster to each RB. Finally, we construct a two-dimensional throughput ${ }_{R j}$ matrix based on the cluster number and the RB number and allocate resources for each cluster according to the constructed matrix.
The throughput ${ }_{R j}$ value of the cluster $g_{k}$ sharing $\mathrm{RB}_{j}$ is defined as

$$
\begin{aligned}
& \text { throughput }_{R j} \\
& =\left[\log _{2}\left(1+\gamma_{\mathrm{CU}}\right)+\sum_{i} \log _{2}\left(1+\gamma_{\mathrm{DU}_{i}}\right)\right], \\
& \gamma_{\mathrm{DU}_{i}}=\frac{P_{i}^{d} h_{d i, d i}}{\sum_{j, l \neq i} P_{l}^{d} h_{d l, d j}+P^{c} h_{c, d i}+N_{0}}, \quad \forall i, l \in g_{k j \text { share }}, \\
& \gamma_{\mathrm{CU}}=\frac{P^{c} h_{c, B}}{\sum_{i} P_{\max }^{d} h_{d i, c}+N_{0}}, \quad \forall i \in g_{k j \text { share }} .
\end{aligned}
$$

Among them, $\gamma_{\mathrm{DU}_{i}}$ is the D2D users' SINR in cluster $g_{k j \text { share }}$ in $\mathrm{RB}_{j}$ and $\gamma_{\mathrm{CU}}$ indicates the cellular users' SINR in $\mathrm{RB}_{j}$ when there is interference from the D2D users in cluster $g_{k j \text { share }}$.

The specific resource allocation process is as follows:

(1) Initialize: according to (11), calculate the throughput $_{R j}$ of each cluster in each $\mathrm{RB}$, and

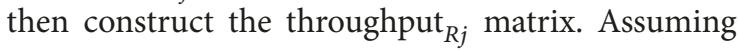
$S$ is the set of RBs that have not yet been allocated, $j$ shows a single $R B, j \in S$.

(2) while $(S \neq \emptyset)$ :

In the throughput ${ }_{R j}$ matrix, find the maximum throughput $_{R j}$ value, and its corresponding $\mathrm{RB}_{j}$ and cluster $g_{k j \text { share; }}$

Allocate $\mathrm{RB}_{j}$ to cluster $g_{k j \text { share }}$;

Set all $\mathrm{RB}_{j}$ corresponding to throughput ${ }_{R j}$ to $-1,000$.

$S=S-\{j\} ;$

End while

(3) Power distribution: the final transmission power of each D2D pair $i$ is

$$
\begin{aligned}
& P_{i}^{d} \\
& =\frac{P_{\max }^{d}}{\left(\text { the number of } \mathrm{RB} \text { that been allocated to } \mathrm{D}_{2} \mathrm{D}_{i}\right)},
\end{aligned}
$$
$P_{\max }^{c}$.

where the transmission power of cellular users is $P^{c}=$

\section{Simulation Results and Analysis}

4.1. Simulation Parameters. A single-cell scenario with a radius of $500 \mathrm{~m}$ is considered, where all CUs and DUs are randomly distributed uniformly in the cell. The D2D pairs and $\mathrm{CU}$ are static, and each receiver and each transmitter have a single antenna. In the simulation, it is assumed that the amount of self-interference cancellation of the full duplex $\mathrm{D} 2 \mathrm{D}$ node is $110 \mathrm{~dB}$ [24], the antenna gain is $14.0 \mathrm{dBi}$ [25], the uplink bandwidth is set to $1.4 \mathrm{MHz}$, and the resource block RB is six. All parameters are shown in Table 1. The 
TABLE 1: Simulation parameters.

\begin{tabular}{lc}
\hline Parameter & Parameter value \\
\hline Radius of cellular users $(\mathrm{Km})$ & 0.5 \\
Average distance of D2D pair $(\mathrm{m})$ & 25 \\
System bandwidth $(\mathrm{MHz})$ & 1.4 \\
RB bandwidth $(\mathrm{KHz})$ & 240 \\
Number of cellular users & 6 \\
Number of D2D pairs & $0,10,20,30,40,50,60,70,80$ \\
The maximum transmit power of cellular user $(\mathrm{dBm})$ & 23 \\
The maximum transmit power of D2D pair $(\mathrm{dBm})$ & 10 \\
Noise spectrum density $(\mathrm{dBm} / \mathrm{Hz})$ & -174 \\
Cellular link road-loss model $(\mathrm{Km})$ [21-23] & $128.1+37.6 \lg D$ \\
D2D link road-loss model $(\mathrm{m})[21-23]$ & $127+30$ lg $D$ \\
Cellular user SINR threshold $(\mathrm{dB})$ & {$[0,25]$ uniform distribution } \\
D2D pair SINR threshold $(\mathrm{dB})$ & {$[0,25]$ uniform distribution } \\
\hline
\end{tabular}

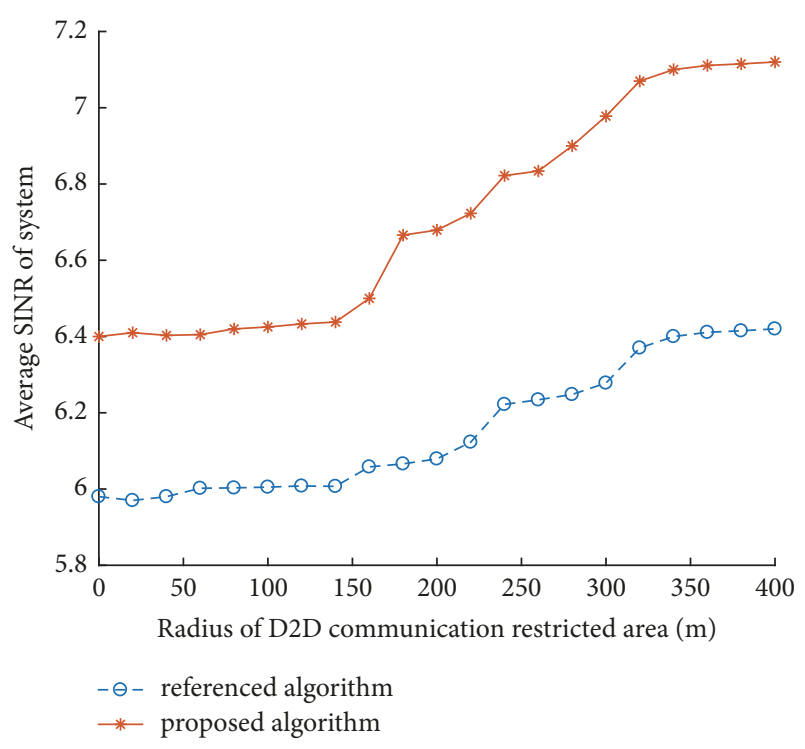

FIGURE 3: Influence of the radius of the D2D communication area versus the average SINR of the system.

simulation was performed using Matlab, where the goal of our proposed scheme is to determine the optimized total system throughput and average SINR, which are then compared with the full duplex random resource allocation scheme of $[18,26]$ to verify its validity.

\subsection{Verification Analysis}

4.2.1. D2D Communication Restricted Area Verification. Figure 3 is a chart of the average SINR change of the system for increasing radii of the D2D communication area. From Figure 3, we can see that as the D2D communication area radius increases from 0 to $150 \mathrm{~m}$, the average SINR increase is not very obvious. However, when the radius exceeds $150 \mathrm{~m}$, the average SINR of the system increases with an increase of the radius of the $\mathrm{D} 2 \mathrm{D}$ communication area. It can be seen that, to reduce the interference from the $\mathrm{D} 2 \mathrm{D}$ users to base

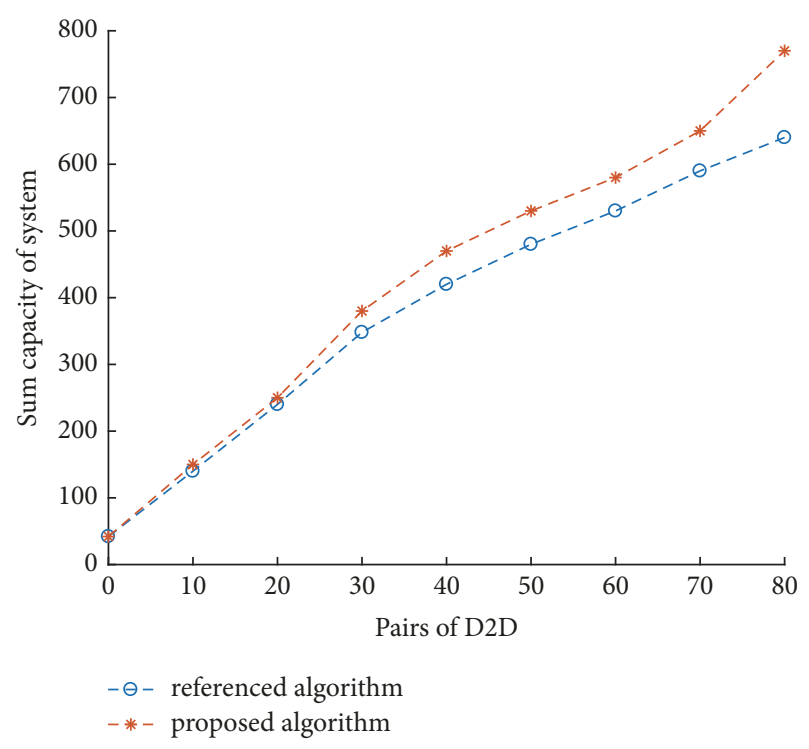

FIGURE 4: Influence of the number of D2D pairs on the total capacity of the system.

station, it is necessary for the cell to delimit a restricted D2D communication area. Under the experimental conditions set in this paper, the radius of the restricted D2D communication area is $\sim 150 \mathrm{~m}$. At the same time, it can be seen from Figure 4 that the proposed algorithm is obviously better than the resource block random allocation algorithm used by other authors $[18,26]$.

4.2.2. Influence of the Number of D2D Pairs to the Total System Throughput Verification. Figure 4 shows the total system throughput of the two resource allocation schemes as the number of D2D pairs increases. From this figure, we can see that when the number of D2D pairs is $<20$, the throughput of the proposed resource allocation scheme is similar to that of the random allocation scheme. As the number of D2D pairs increases, the total throughput of the two schemes gradually increases, but the throughput of our 


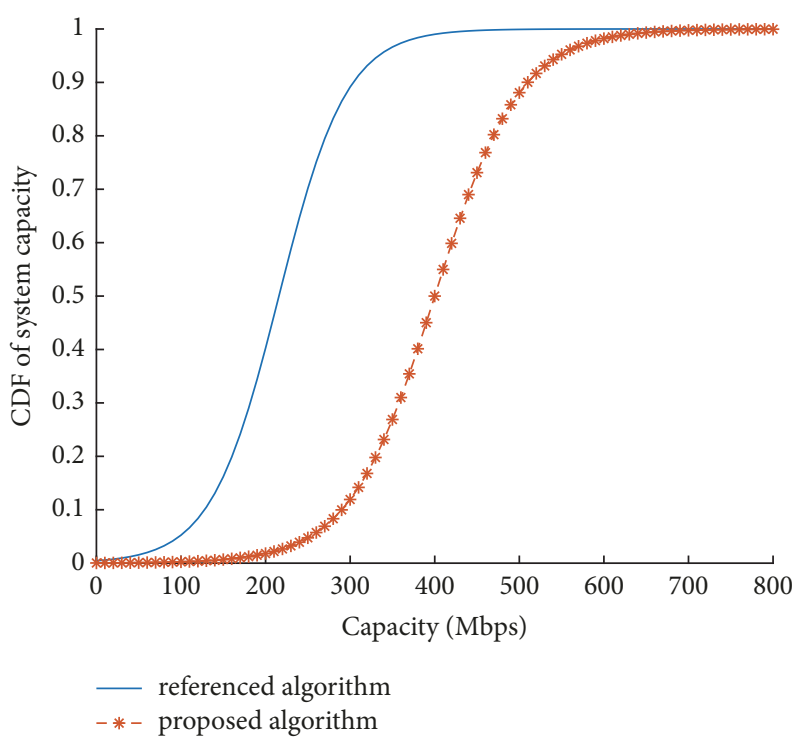

FIGURE 5: CDFs of the system capacity for the two considered schemes.

proposed scheme is significantly higher than that of the random resource allocation scheme.

4.2.3. Comparison of the Cumulative Distribution of the Total System Throughput. Figure 5 shows the cumulative distribution functions (CDFs) of the total throughput of the system for the two schemes. Since our method uses a $K$ means algorithm to cluster the D2D pairs, where interference can be ignored for each pair, and then allocates the optimal $\mathrm{RB}$ to the D2D cluster, we are able to fully utilize the system's available and reusable RBs, and the number of D2D links established in the cellular system is maximized. Therefore, compared with the reference algorithm, the total throughput of the system has been significantly improved.

\section{Conclusions}

We analyzed the status of D2D pairs that reuse the resources of cellular users in the same $5 \mathrm{G}$ channel in a cellular communication system to eliminate the interference of D2D pairs to the base station, CU users to $\mathrm{D} 2 \mathrm{D}$ receivers, and D2D pairs with each other. We proposed several countermeasures and solutions, where a function model was constructed to maximize the system's efficiency. To eliminate the interference of D2D users to the base station, the concept of a limited $\mathrm{D} 2 \mathrm{D}$ communication area is proposed. The interference of D2D users to the base station outside of the restricted area is negligible. The concept of a limited reusage area to combat the interference of CU users to D2D users is proposed. It is possible for the $\mathrm{D} 2 \mathrm{D}$ users to reuse the $\mathrm{CU}$ resource outside of the restricted area. To address the interference between $\mathrm{D} 2 \mathrm{D}$ pairs, we suggest using the interference intensity value to the cluster D2D pairs based on a $K$-means algorithm, where the larger the interference value is between D2D pairs, the smaller the probability of being placed into a cluster is. In the same cluster, the interference can be neglected between D2D pairs; that is to say, they can reuse the same CU resources. Then, we described the resource allocation algorithm, where we allocated CU resource blocks to the clustered D2D pairs and allocated the best RBs to D2D pairs. As demonstrated with a simulation performed with Matlab, our method is able to eliminate interference and improve the system's overall performance.

\section{Data Availability}

The data used to support the findings of this study are available from the corresponding author upon request.

\section{Conflicts of Interest}

The authors declare that they have no conflicts of interest.

\section{Acknowledgments}

This work was partly supported by the National Natural Science Foundation of China (61202290, 61370173, and 61772198).

\section{References}

[1] J. F. Monserrat, G. Mange, V. Braun, H. Tullberg, G. Zimmermann, and Ö. Bulakci, "METIS research advances towards the 5G mobile and wireless system definition," EURASIP Journal on Wireless Communications and Networking, vol. 2015, no. 1, article 53, 2015.

[2] K. Doppler, M. Rinne, C. Wijting, C. B. Ribeiro, and K. Hug, "Device-to-device communication as an underlay to LTEadvanced networks," IEEE Communications Magazine, vol. 47, no. 12, pp. 42-49, 2009.

[3] J. Du, W. Zhu, J. Xu, Z. Li, and H. Wang, "A compressed HARQ feedback for device-to-device multicast communications," in Proceedings of the IEEE Vehicular Technology Conference (VTCFall '12), pp. 1-5, IEEE, Quebec City, Canada, September 2012.

[4] Z. Li, M. Moisio, M. Uusitalo et al., "Overview on initial METIS D2D Concept," in Proceedings of the 1st International Conference on 5G for Ubiquitous Connectivity, pp. 203-208, IEEE Press, Levi, Finland, November 2014.

[5] R. Tang, J. Zhao, H. Qu, Z. Zhu, and Y. Zhang, "Joint mode selection and resource allocation for mobile relay-aided deviceto-device communication," KSII Transactions on Internet and Information Systems, vol. 10, no. 3, pp. 950-975, 2016.

[6] S. Ali, N. Rajatheva, and M. Latva-Aho, "Full duplex deviceto-device communication in cellular networks," in Proceedings of the 2014 European Conference on Networks and Communications, EuCNC 2014, pp. 1-5, Bologna, Italy, June 2014.

[7] X. Yao, X. Xie, and Y. Tian, "A new interference alignment algorithm for device-to-device communication underlaying multi-cell MIMO networks," Journal of Chongqing University of Posts and Telecommunications (Natural Science Edition, vol. 28, no. 5, pp. 641-647, 2016.

[8] Z. Zhang, K. Long, A. V. Vasilakos, and L. Hanzo, "Fullduplex wireless communications: challenges, solutions, and future research directions," Proceedings of the IEEE, vol. 104, no. 7, pp. 1369-1409, 2016. 
[9] R. Tang, J. Zhao, H. Qu, and Z. Zhang, "Energy-efficient resource allocation for $5 \mathrm{G}$ full-duplex enabled device-to-device communication," in Proceedings of the 1st IEEE Global Communications Conference on Full Duplex Wireless Communications International Workshop, pp. 1-7, IEEE Press, Washington, DC, USA, December 2016.

[10] G. Fodor and N. Reider, "A distributed power control scheme for cellular network assisted D2D communications," in Proceedings of the IEEE Global Telecommunications Conference (GLOBECOM '11), pp. 1-6, Houston, Tex, USA, December 2011.

[11] L. Song, D. Niyato, Z. Han, and E. Hossain, "Game-theoretic resource allocation methods for device-to-device communication," IEEE Wireless Communications Magazine, vol. 21, no. 3, pp. 136-144, 2014.

[12] L. Yang, S. He, Y. Wang et al., "Key technologies for 5G wireless communication system," Journal of Data Acquisition \& Processing, vol. 30, no. 3, pp. 469-485, 2015.

[13] A. H. Sakr, H. Tabassum, E. Hossain, and D. I. Kim, "Cognitive spectrum access in device-to-device-enabled cellular networks," IEEE Communications Magazine, vol. 53, no. 7, pp. 126-133, 2015.

[14] T. E. Bogale and L. Vandendorpe, "Linearly combined signal energy based spectrum sensing algorithm for cognitive radio networks with noise variance uncertainty," in Proceedings of the 2013 8th International Conference on Cognitive Radio Oriented Wireless Networks and Communications, CROWNCOM 2013, pp. 80-86, IEEE Press, Washington, DC, USA, July 2013.

[15] L. Tong, Y. Liu, J. Lou, J. Lu, and F. E. Alsaadi, "Static output feedback set stabilization for context-sensitive probabilistic Boolean control networks," Applied Mathematics and Computation, vol. 332, pp. 263-275, 2018.

[16] Y. Liu, L. Y. Tong, J. G. Lou et al., "Sampled-data control for the synchronization of boolean control networks," IEEE Transactions on Cybernetics, pp. 1-7, 2018.

[17] J. Lou, Y. Jiang, Q. Shen, Z. Shen, Z. Wang, and R. Wang, "Software reliability prediction via relevance vector regression," Neurocomputing, vol. 186, pp. 66-73, 2016.

[18] N. Chen, H. Tian, and Z. Wang, "Resource allocation for intra-cluster D2D communications based on Kuhn-Munkres algorithm," in Proceedings of the 80th IEEE Vehicular Technology Conference, VTC 2014-Fall, pp. 1-5, September 2014.

[19] Y. Liu, Y. Xu, D. Li, and W. Wang, "Device-to-Device communication in LTE-A cellular networks: Standardization, architecture, and challenge," in Proceedings of the 2014 IEEE Vehicular Technology Conference (VTC 2014-Spring), pp. 1-5, IEEE, Seoul, South Korea, May 2014.

[20] X. Lin, J. G. Andrews, A. Ghosh, and R. Ratasuk, "An overview of 3GPP device-to-device proximity services," IEEE Communications Magazine, vol. 52, no. 4, pp. 40-48, 2014.

[21] C. Lee, S. Oh, and A. Park, "Interference avoidance resource allocation for D2D communication based on graph-coloring," in Proceedings of the 2014 International Conference on Information and Communication Technology Convergence (ICTC), pp. 895-896, Busan, South Korea, October 2014.

[22] J. Han, Q. Cui, C. Yang, and X. Tao, "Bipartite matching approach to optimal resource allocation in device to device underlaying cellular network," IEEE Electronics Letters, vol. 50, no. 3, pp. 212-214, 2014.

[23] D. Zhu, J. Wang, A. L. Swindlehurst, and C. Zhao, "Downlink resource reuse for device-to-device communications underlaying cellular networks," IEEE Signal Processing Letters, vol. 21, no. 5, pp. 531-534, 2014.
[24] G. Zhu, T. Liu, and J. Yang, "Optimal power control for 5G fullduplex enabled D2D communication," Application Research of Computers, vol. 34, no. 12, pp. 1-5, 2017.

[25] R. Tang, J. Dang, X. Zhuang et al., “Throughput-capacity tradeoff for device-to-device communication underlaying cellular net-work via joint resource allocation," in Proceedings of the International Conference on Communication Technology, pp. 181-190, IEEE Press, 2015.

[26] D. J. Son, C. H. Yu, and D. I. Kim, "Resource allocation based on clustering for D2D communications in underlaying cellular networks," in Proceedings of the 5th International Conference on Information and Communication Technology Convergence, ICTC 2014, pp. 232-237, October 2014. 


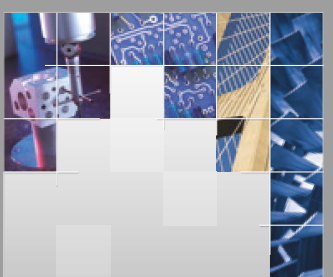

\section{Enfincering}
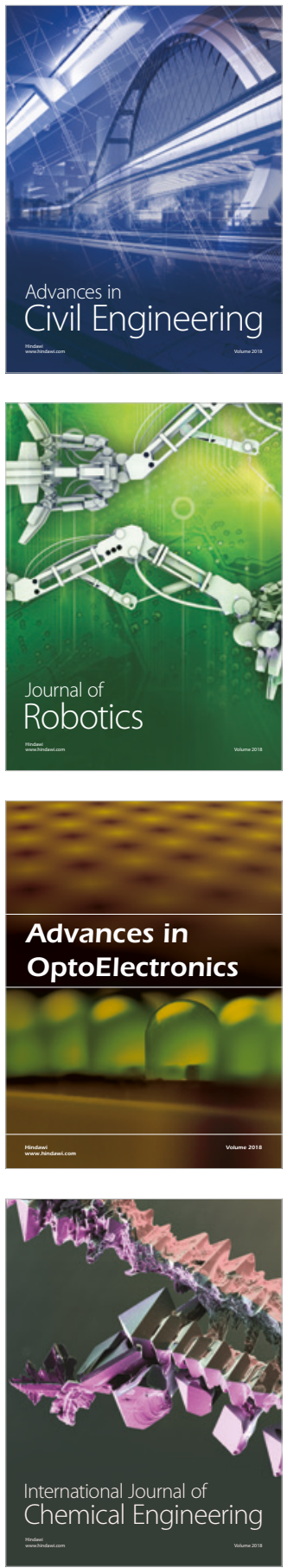

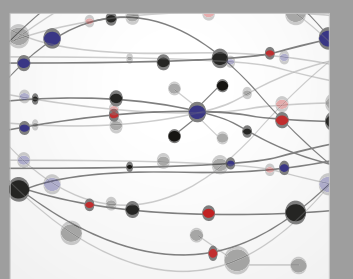

\section{Rotating \\ Machinery}

The Scientific World Journal

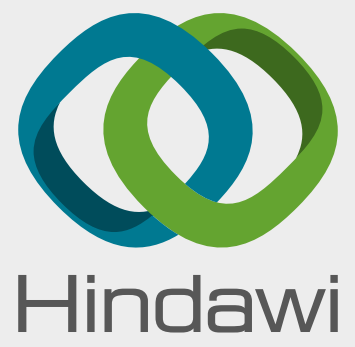

Submit your manuscripts at

www.hindawi.com
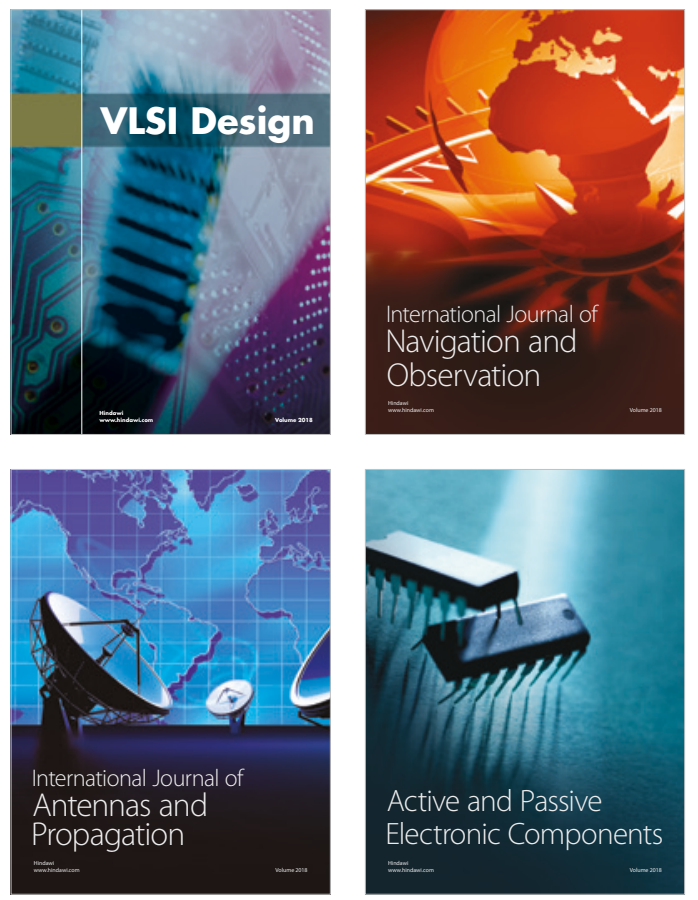
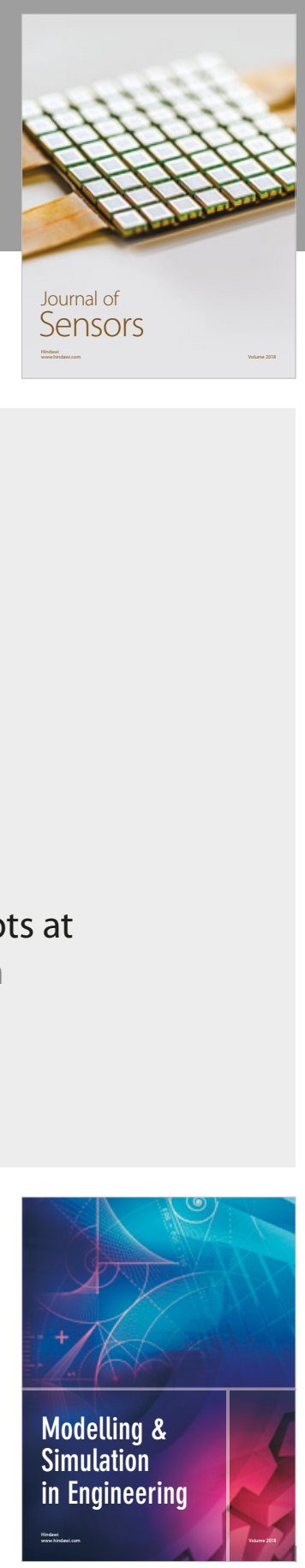

\section{Advances \\ Multimedia}
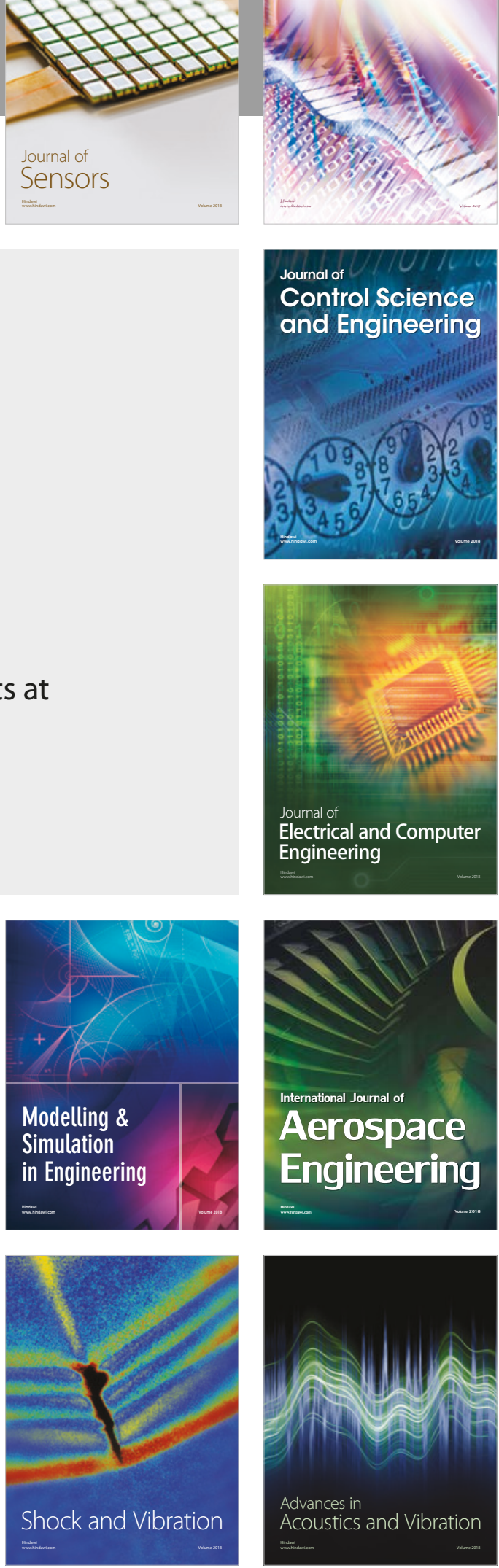